\title{
APPROXIMATION OF CONTINUOUS FUNCTIONS BY INTEGRAL FUNCTIONS OF FINITE ORDER
}

\author{
BY \\ H. KOBER
}

In the proof of his classical theorem on approximation by polynomials Weierstrass first constructs integral functions of the order two, to approximate to a continuous function uniformly over a finite in terval. The more general problem: Which condition is necessary and sufficient for a function $f(x)$ to be approximated by integral functions uniformly over $(-\infty, \infty)$ ? was solved by $\mathrm{T}$. Carleman( $\left.{ }^{1}\right)$, and a slight refinement of his method gives the following interesting result:

(A) Given any positive increasing function $\phi(x)(0 \leqq x<\infty)$, there are integral functions $g_{n}(z)(z=x+i y ; n=1,2, \cdots)$ such that

$$
\underset{-\infty<x<\infty}{\text { u.b. } \phi(|x|)\left|f(x)-g_{n}(x)\right| \rightarrow 0 \quad \text { as } n \rightarrow \infty,}
$$

provided only that $f(x)$ is continuous in $(-\infty, \infty)$.

The function $f(x)$ need not be bounded. On the other hand, nothing can be stated about the nature of the $g_{n}(z)$. These functions may be integral functions of infinite order; take, for instance, $f(x)=x^{1 / 2} \exp \left(e^{x}\right)$. To obtain functions of finite order, stronger conditions are to be imposed on $f(x)$.

Let first $f(x)$ be supposed to be bounded. By known results $\left({ }^{2}\right)$ the condition should be the weaker, the higher the order of $g_{n}(z)$ is, and the problem is answered by the following theorem (stated without proof):

(B) When $\rho$ is a positive integer and $\omega \geqq-\infty$ then $f(x)$ is approximated, uniformly in $(\omega, \infty)$, by functions $g_{\alpha}(x) \in G_{\alpha}^{(\rho)}(0<\alpha \rightarrow \infty)$ if, and only if, $\psi(x)$ is uniformily continuous in $\left(\left|\omega^{\rho}\right| \operatorname{sgn} \omega, \infty\right)$, where $\psi(x)=f\left(\left|x^{1 / \rho}\right| \operatorname{sgn} x\right)$ for $x \geqq \omega$.

This result is known for $\omega=-\infty, \rho=1\left({ }^{2}\right)$. The necessity of the condition is deduced from the following theorem (stated without proof):

(C) If $1 / 2 \leqq \rho<\infty, g_{\alpha}(z) \in G_{\alpha}^{(\rho)}(0<\alpha<\infty)$, and if $g_{\alpha}(x)$ is bounded in $(0, \infty)$, then $x^{1-\rho} g_{\alpha}^{\prime}(x)$ is bounded in $(1, \infty)$, and so $g_{\alpha}\left(x^{1 / \rho}\right)$ is uniformly continuous in $(0, \infty)$.

Presented to the Society, December 29, 1946; received by the editors May 13, 1946.

(1) T. Carleman, Arkiv för Matematik, Astronomi och Fysik vol. 20 B (1927).

(2) H. Kober, Trans. Amer. Math. Soc. vol. 54 (1943) pp. 70-82, and vol. 56 (1944) pp. 731. References to these papers are indicated by $F$ and $G$, respectively. See Theorems $2 F$ and $3 G$, and, for the case $\rho=1, \omega=-\infty, 3(\mathrm{~b}) \mathrm{F}$. The set of in tegral functions $F(z)$, such that, for any $\epsilon>0$ and for some $\alpha(0 \leqq \alpha<\infty),|F(z)|<A_{\epsilon} \exp \{(\alpha+\epsilon)|z| \rho\}$, is denoted by $G_{\alpha}^{(\rho)}$, and $G_{\alpha}$ is written for $G_{\alpha}^{(1)}$. Evidently $G_{\alpha}^{(\sigma)} \subset G_{\beta}^{(\rho)}$ when $0<\sigma<\rho$. Notice that, for $0<\rho<\lambda<\mu<\infty$, the uniform continuity of $f\left(x^{\lambda}\right)$ in $(0, \infty)$ implies that of $f\left(x^{\rho}\right)$ in $(0, \infty)$, but that $f\left(x^{\mu}\right)$ on any finite interval only. 
This can be considered as a generalization of Bernstein's theorem (see §2 of the present paper).

The condition in Theorem (B) can be shown to suffice even if $f(x)$ is not bounded; and possibly this result holds whenever $1 / 2 \leqq \rho<\infty$, with $\omega>-\infty$ for $1 / 2 \leqq \rho<1$.

Now approximation shall be considered by elements of $G_{\alpha}$ only. The function $f(x)$ shall not be required to be bounded in the familiar sense; but, from the above remark to (A), it is evident that some restriction of a related kind is necessary. The cases when $f(x)$ is represented asymptotically (as $x \rightarrow \pm \infty$ ) by linear functions or satisfies a Lipschitz condition or a condition of the Taylor type have been investigated $\left(^{(3}\right)$. In the present paper a much more general case is treated, and under that weaker condition the degree of approximation is considerably improved corresponding to (A) (see 3.11, 3.12, 4.12). This justifies the basic definitions given in $\$ 1$. To deal with necessary conditions, a basic property of integral functions of exponential type is deduced (Theorem 2). The results of $\$ 3$ are extended to functions analytic in the half-plane in $\$ 4$.

Non-negative integers will be denoted by $j, l, m, n$, real numbers by $h, k$, positive numbers by $A, B, H, K$.

We write

$$
\Delta_{n, h} f(x)=\sum_{j=0}^{n}(-1)^{j+n} C_{n, j} f(x+j h) ; \Delta_{0, h} f(x)=f(x) .
$$

1. Basic definitions and lemmas. Elementary properties of functions, which are uniformly bounded or uniformly continuous of order $n$, will now be discussed.

\subsection{Definitions.}

(I) A function $f(x)$ is said to be uniformly bounded of order $n$ if (i) it is bounded in some interval (no matter how small), and if (ii) there are two numbers $H$ and $K$ such that, uniformly for $-\infty<x<\infty,\left|\Delta_{n, h} f(x)\right| \leqq K$ whenever $-H \leqq h \leqq H$. When $n=0$ then $f(x)$ is uniformly bounded in the familiar sense.

(II) A function $f(x)$ is said to be uniformly continuous of order $n(n \geqq 1)$ if (i) it satisfies the condition (i) of (I) and is (ii) measurable on any finite interval, and if (iii), given $\epsilon>0$, there is $a \delta=\delta(\epsilon)>0$ such that, uniformly for $-\infty<x<\infty$, $\left|\Delta_{n, h} f(x)\right| \leqq \epsilon$ whenever. $-\delta \leqq h \leqq \delta$.

It is evident that, for $n=0$ and $n=1$, the condition (i) is omitted in definition (I); so are (i) and (ii) in (II) for $n=1$. The property (II) of a function implies (I). The converse is, in general, not true; the function $\sin x^{2}$, for example, is uniformly bounded of any order, but there is no order of which it is uniformly continuous. It is, however, true for the class $G_{\alpha}$ (see $\$ 2$ ).

Example (i). The function $x^{r}$ (arg $x=0$ or arg $x=\pi$ for $x>0$ or $x<0$, respectively, $n<r \leqq n+1)$ is uniformly bounded and uniformly continuous of order $n+1$, but not of any smaller order.

(3) Theorem 3(c)F and the Bernstein Theorem 3(d)F; Theorem 10G. 
This follows from the equalities (cf. G, p. 22, line 6)

$$
\begin{aligned}
\Delta_{m, h} x^{r} & =m ! C_{m, r} \int_{0}^{h} \cdots \int_{0}^{h}\left(x+t_{1}+t_{2}+\cdots+t_{m}\right)^{r-m} d t_{1} d t_{2} \cdots d t_{m} ; \\
\Delta_{n+1, h} x^{r} & =\Delta_{n, h}(x+h)^{r}-\Delta_{n, h} x^{r} ;\left|\Delta_{1, h} x^{r-n}\right| \leqq 2^{1-r+n}|h|^{r-n} .
\end{aligned}
$$

Example (ii). $f(x)=x^{r}(x+\alpha)^{-s} ; s \geqq 0$ or $s<0,0 \leqq r \leqq n+s+1 ; \Im(\alpha) \neq 0$.

This function is uniformly continuous of order $n+1$, or of some smaller order.

Example (iii). $f(x)=\log \left(\sum_{j=0}^{n} \alpha_{j} x_{j}\right), r_{j} \geqq 0 ; \sum_{j=0}^{n} \alpha_{j} x^{r_{j}} \neq 0$ for $-\infty<x<\infty$. This function is uniformly continuous (of order one).

Example (iv). $f(x)=x \sin \left|x^{1 / 2}\right|$ is uniformly continuous of order two, but of no smaller order.

Example (v). If $f(x)$ is uniformly continuous of order $n$ then the same is true for the function (cf. \$3.2), belonging to $G_{\alpha}$,

$$
g_{\alpha}(x)=\int_{-\infty}^{\infty}\left(\frac{\sin t /(2 n+2)}{t}\right)^{2 n+2} f(x+t / \alpha) d t \quad(\alpha>0) .
$$

1.2. Some lemmas, and a theorem.

Lemma 1.

$$
\begin{aligned}
\Delta_{n,-h} f(x) & =(-1)^{n} \Delta_{n, h} f(x-n h) & & (n \geqq 0), \\
\Delta_{n, h} f(x) & =\Delta_{n-1, h} f(x+h)-\Delta_{n-1, h} f(x) & & (n \geqq 1),
\end{aligned}
$$

and, writing $\Delta_{m, k}\left\{\Delta_{n, h} f(x)\right\}=\Delta_{m, k} \Delta_{n, h} f(x)$, we have

$$
\Delta_{m, k} \Delta_{n, h} f(x)=\Delta_{n, h} \Delta_{m, k} f(x) .
$$

LEMma 2. If $\left|\Delta_{n, h} f(x)\right| \leqq K$ for $|h| \leqq H$, then

$$
\left|\Delta_{m, k} \Delta_{n, h} f(x)\right| \leqq 2^{m} K \quad(|h| \leqq H,-\infty<k<\infty,-\infty<x<\infty) .
$$

CoRollary. If $f(x)$ is uniformly bounded of order $n$, then it is uniformty bounded of any higher order. The result holds for uniform continuity of order $n(n \geqq 1)$.

These lemmas are evident.

Lemma 3.

$$
\Delta_{n, 2 h} f(x)=\sum_{j=0}^{n} C_{n, j} \Delta_{n, h} f(x+j h) .
$$

This is shown by means of the equations

$$
\sum_{j=0}^{n}(-1)^{m-j} C_{n, j} C_{n, m-j}=\left\{\begin{array}{cc}
(-1)^{m / 2} C_{n, m / 2} & (m \text { even }), \\
0 & (m \text { odd }),
\end{array}\right.
$$




$$
\begin{aligned}
\sum_{j=0}^{n}(-1)^{l} C_{n, l} f(x+2 l h) & =\sum_{m=0}^{2 n} f(x+m h) \sum_{j=0}^{n}(-1)^{m-i} C_{n, j} C_{n, m-i} \\
& =\sum_{j=0}^{n} C_{n, j} \sum_{j_{1}=0}^{n}(-1)^{j 1} C_{n, j_{1}} f\left\{x+\left(j+j_{1}\right) h\right\}\left(j_{1}=m-j\right) \\
& =\sum_{j=0}^{n} C_{n, j}(-1)^{n} \Delta_{n, h} f(x+j h) .
\end{aligned}
$$

THEOREM 1. If $f(x)$ is uniformly continuous of order $n$, and if $f(x)$ is uniformly-bounded of order $m(m<n)$, then $f(x)$ is uniformly continuous of order $m$ when $m \geqq 1$, of order 1 when $m=0$.

This result appears to be elementary, yet we are unable to give an elementary proof. Anticipating the Theorems 3 (for $m=0$ ) and 2, we see that the functions $g_{\alpha}(x)$, approximating to $f(x)$, are uniformly bounded of order $m$ and, therefore, uniformly continuous of order $\max (1, m)$. This gives the result required. In a similar way we can show:

THEOREM $1^{\prime}$. If $f(x)$ is uniformly continuous of order $n(n>1)$, then it is continuous in the familiar sense on any finite interval.

\subsection{Two estimates.}

LEMma 4. If $\left|\Delta_{n, t} f(x)\right| \leqq K$ for $-H \leqq t \leqq H$, then

$$
\left|\Delta_{n, t} f(x)\right| \leqq(2 / H)^{n} K|t|^{n} \quad \text { for } t \geqq H \text { and for } t \leqq-H \text {. }
$$

Proof. Let $t>H, 2^{l} H<t \leqq 2^{l+1} H, h=t / 2^{l+1}$. Then $0<h \leqq H$. By Lemma 3 ,

$$
\left|\Delta_{n, 2 h} f(x)\right| \leqq 2^{n} \max _{j=0,1, \cdots, n}\left|\Delta_{n, h} f(x+j h)\right| \leqq 2^{n} K .
$$

Repeating this procedure, we have finally

$$
\left|\Delta_{n, t} f(x)\right|=\left|\Delta_{n, 2 l+1 h} f(x)\right| \leqq 2^{(l+1) n} K \leqq(2 t / H)^{n} K,
$$

as $2^{l}<t / H$. In a similar way we proceed when $t<-H$.

Remark. Evidently for $-\infty<t<\infty,-\infty<x<\infty$ we have

$$
\left|\Delta_{n, t} f(x)\right| \leqq A\left(1+|t|^{n}\right) \text {. }
$$

LEMMA 5. If $f(x)$ is uniformly bounded of order $n$, then

$$
|f(x)| \leqq A\left(1+|x|^{n}\right) \text {. }
$$

Proof. It is obvious that for $-\infty<\xi<\infty ; j_{1}=0,1,2, \cdots$,

$$
\Delta_{n-1, h} f\left(\xi+j_{1} h\right)=\Delta_{n-1, h} f(\xi)+\sum_{j=0}^{j-j_{1}-1} \Delta_{n, h} f(\xi+j h),
$$

the latter sum vanishing when $j_{1}-1<0$, that is, when $j_{1}=0$. Hence 


$$
\begin{aligned}
\Delta_{n-m, h} f\left(x+j_{m} h\right) & =\sum_{j=0}^{m-1} C_{j_{m}, j} \Delta_{n-m+j, h} f(x) \\
+\sum_{j_{m-1}=0}^{j_{m-1=j_{m}-1}} & \sum_{j_{m-2}=0}^{j_{m-2=}=j_{m-1}-1} \cdots \sum_{j=0}^{j=j_{1}-1} \Delta_{n, h} f(x+j h) \quad(1 \leqq m \leqq n) .
\end{aligned}
$$

By definition, $f(x)$ is bounded in some interval; we may, therefore, suppose that it is bounded in $(-n H, n H)$. Let now $m=n, y>n H$ or $y<-n H$; $l H<|y| \leqq(l+1) H ; h=(l+1)^{-1} y ; j_{n}=j_{m}=l+1$. Then $j_{n} \leqq H^{-1}|y|+1,|h| \leqq H$ and, by hypothesis, $\left|\Delta_{n, h} f(j h)\right| \leqq K$. Thus, taking $x=0$ in (1.34), we have

$$
\begin{aligned}
& |f(y)|=\left|f\left(j_{n} h\right)\right| \leqq n j_{n}^{n-1} \max _{j=0,1, \cdots, n-1}\left|\Delta_{j, h} f(0)\right| \\
& \quad+j_{n}^{n} \max _{j=0,1, \cdots, j_{n}-1}\left|\Delta_{n, h} f(j h)\right| \leqq B j_{n}^{n-1}+K j_{n}^{n} \leqq A^{\prime}\left(H^{-1}|y|+1\right)^{n},
\end{aligned}
$$

which proves the lemma.

2. A basic property of integral functions of exponential type. Now integral functions of exponential type, which are uniformly bounded of order $n$, will be dealt with.

\subsection{The result, and some lemmas,}

LEMMA 6 (BeRnSTEIN's THEOREM( $\left.{ }^{4}\right)$ ). If $f(z)$ belongs to $G_{\alpha}(\alpha>0)$, and if $|f(x)| \leqq A$ for $-\infty<x<\infty$, then $\left|f^{\prime}(x)\right| \leqq \alpha A$ for $-\infty<x<\infty$.

THEOREM 2. If $f(z)$ belongs to $G_{\alpha}(\alpha>0)$, and if $f(x)$ is uniformly bounded of order $n(n \geqq 1)$, then $f^{(n)}(x)$, its derivative of order $n$, is uniformly bounded in $(-\infty, \infty)$ in the familiar sense, and $f(x)$ is uniformly continuous of order $n$.

THEOREM 2 '. If $f(z)$ belongs to $G_{0}$, and if $f(x)$ is uniformly bounded of order $n(n \geqq 0)$, then $f(z)$ reduces to a polynomial of degree not greater than $n$.

CoRollary. When $f(z)$ is an integral function and $f(x)$ is uniformly bounded of order $n$, then either $f(z)$ is a polynomial of degree not greater than $n$, or there are points $z_{1}, z_{2}, \cdots\left(\left|z_{j}\right| \rightarrow \infty\right)$ and a constant $\alpha>0$ such that $\left|f\left(z_{j}\right)\right|>e^{\alpha\left|z_{j}\right|}(j=1,2, \cdots)$.

Lemma 7. If $\Phi(x)$ is uniformly bounded of order $m$, and $\Phi^{(m)}(x)$ is an integral, and if $\Phi^{(m+1)}(x)$ is uniformly bounded in the familiar sense, then $\Phi^{(m)}(x)$ is uniformly bounded in this sense as well.

2.2. Proof of Lemma 7, and of Theorem 2 for $n=1\left(^{5}\right)$. Without loss of gener-

(') S. Bernstein, Leçons sur les propriêtés extrémales des fonctions analytiques d'une variable reelle, Paris, 1926, p. 102, corollary.

(5) As the referee pointed out to me, this can alternatively be deduced from a result of Duffin and Schaeffer, Amer. J. Math. vol. 67 (1945) p. 141; This is also true for $n>1$. 
ality we may suppose that $\Phi(x)$ and $f(x)$ are real-valued. Let $\left|\Delta_{m, h} \Phi(x)\right| \leqq K$ for $|h| \leqq H$, and $\left|\Phi^{(m+1)}(x)\right| \leqq B$ in $(-\infty, \infty)$. If, for some $x, \Phi^{(m)}(x)$ were greater than $A=H B+m^{m} H^{-m} K$, we should have

$$
\begin{aligned}
\Phi^{(m)}\left(x+t_{1}+t_{2}+\cdots+t_{m}\right) & =\Phi^{(m)}(x)+\int_{0}^{t_{1}+t_{2}+\cdots+t_{m}} \Phi^{(m+1)}(x+t) d t \\
& >H B+m^{m} H^{-m} K-H B=m^{m} H^{-m} K,
\end{aligned}
$$

for $0 \leqq t_{j} \leqq m^{-1} H(j=1,2, \cdots, m)$. Thus we should arrive at the contradiction

$$
\begin{aligned}
\Delta_{m, h} \Phi(x) & =\int_{0}^{h} \int_{0}^{h} \cdots \int_{0}^{h} \Phi^{(m)}\left(x+t_{1}+t_{2}+\cdots+t_{m}\right) d t_{1} d t_{2} \cdots d t_{m} \\
& >h^{m} m^{m} H^{-m} K=K,
\end{aligned}
$$

for $h=m^{-1} H$. This proves the lemma.

Proof of the theorem for $n=1$. By Bernstein's theorem, $|f(x+h)-f(x)|$ $\leqq K$ for $|h| \leqq H$ implies that

$$
\left|f^{\prime}(x+h)-f^{\prime}(x)\right| \leqq \alpha K \quad(-\infty<x<\infty,-H \leqq h \leqq H) .
$$

If, for some $x, f^{\prime}(x)$ were greater than $K / H+\alpha K$ then, using (2.21), we should have the contradiction

$$
\begin{aligned}
f(x+H)-f(x) & =\int_{0}^{B} f^{\prime}(x+h) d h \geqq \int_{0}^{B} f^{\prime}(x) d h \\
& -\int_{0}^{B}\left|f^{\prime}(x+h)-f^{\prime}(x)\right| d h>B(K / H+\alpha K)-\alpha H K=K .
\end{aligned}
$$

Therefore $\left|f^{\prime}(x)\right| \leqq K(1 / H+\alpha)$, which proves the theorem for $n=1$. Probably in the general case we should have $\left|f^{(n)}(x)\right| \leqq A_{n} K(1 / H+\alpha)^{n}(-\infty<x<\infty)$ where $A_{n}$ depends on $n$ only.

2.3. Proof of the theorem for $n>1$. Suppose that the theorem is true for $n^{\prime}=n-1$. It is not difficult to show that

$$
(-1)^{n} \Delta_{n, k-h} f(x+n h)=\sum_{j=0}^{n}(-1)^{i} C_{n, j} \Delta_{n-i, h} \Delta_{i, k} f(x) .
$$

Let $0<h \leqq H, 0<k \leqq H$. By the hypothesis, the moduli of the terms

$$
\Delta_{n, k-h} f(x+n h) ; \quad \Delta_{n, h} \Delta_{0, k} f(x)=\Delta_{n, h} f(x) ; \quad \Delta_{0, h} \Delta_{n, k} f(x)
$$

do not exceed $K$. Hence the modulus of

$$
\sum_{j=1}^{n-1}(-1)^{i} C_{n, j} \Delta_{n-j, h} \Delta_{j, k} f(x)=g_{h, k}(x)
$$


is not greater than $3 K$. For $n=2$, this gives

$$
\left|\Delta_{1, h} \Delta_{1, k} f(x)\right| \leqq 3 K / 2 \text {. }
$$

If $n>2$ we apply the operator $\Delta_{1, h}$ to (2.32). By Lemma 2, the modulus of

$$
\Delta_{1, h} \Delta_{n-1, h} \Delta_{1, k} f(x)=\Delta_{1, k} \Delta_{n, h} f(x)
$$

is not greater than $2 K$. Hence we have

$$
\sum_{j=2}^{n-1}(-1)^{i} C_{n, j} \Delta_{n+1-i, h} \Delta_{j, k} f(x)=\bar{g}_{h, k}(x),
$$

where $\left|\bar{g}_{h, k}(x)\right| \leqq 2 K(n+3)$. Repeating this procedure $(n-3)$ times, we have finally

$$
\left|\Delta_{n-1, h} \Delta_{n-1, k} f(x)\right| \leqq A_{n} K,
$$

where $A_{n}$ depends on $n$ only. Now the theorem holds for $n^{\prime}=n-1$, and we have $\Delta_{n-1, k} f(z) \in G_{\alpha}$. Consequently

$$
\left|\frac{d^{n-1}}{d x^{n-1}} \Delta_{n-1, k} f(x)\right|=\left|\Delta_{n-1, k} f^{(n-1)}(x)\right| \leqq A^{\prime} \quad(0<k \leqq H,-\infty<x<\infty),
$$

and applying the theorem again, we have

$$
\left|f^{(2 n-2)}(x)\right| \leqq A^{\prime \prime} \quad(-\infty<x<\infty) .
$$

If $n=2$ the proof is complete. If $n>2$ then $2 n-2>n$; by the corollary to Lemma $2, f(x)$ is uniformly bounded of order $m=2 n-3$. Now we make use of Lemma 7 , and we deduce that $\left|f^{(2 n-3)}(x)\right| \leqq A^{\prime \prime \prime}$. Repeating this procedure $(n-3)$ times, we arrive at the result required

$$
\left|f^{(n)}(x)\right| \leqq A \quad(-\infty<x<\infty) .
$$

The uniform continuity (order $n$ ) of $f(x)$ is now easily deduced.

2.4. Proof of Theorem 2'. The result can be deduced from Theorem 2; but, as the referee pointed out to me, more directly from a known result, using Lemma $5\left(^{(}\right)$. If $f(z) \in G_{0}$ and $|f(x)| \leqq A\left(1+|x|^{m}\right)$, then $f^{(m)}(x)$ is constant in $(-\infty, \infty)$; for the function $g(z)=z^{-m}\left(f(z)-\sum_{j=0}^{m} z^{i} f^{i}(0) / j !\right)$ belongs to $G_{0}$ and is bounded on the real axis $(-\infty<x<\infty)$, and reduces, therefore; to a constant.

3. Uniform approximation by integral functions of exponential type. In this section the main results will be proved.

\subsection{Statement of the results.}

THEOREM 3. Let $f(x)$ be uniformly bounded of order $n$, and let the nonnegative integer $m$ be fixed. A necessary and sufficient condition for the existence of functions $g_{\alpha}(z) \in G_{\alpha}(0<\alpha<\infty)$ such that

(6) Cf. R. P. Boas, Ann. of Math. vol. 39 (1938) p. 279. 


$$
\underset{-\infty<x<\infty}{\text { u.b. }}\left|f(x)-(x+i)^{m} g_{\alpha}(x)\right| \rightarrow 0
$$

as $\alpha \rightarrow \infty$ is the uniform continuity (order $n$ ) of $f(x)$.

CoRollary (i). If $(x+i)^{m} f(x)$ is uniformly continuous of some order, then there are functions $g_{\alpha}(z) \in G_{\alpha}(0<\alpha<\infty)$ such that

$$
\underset{-\infty<x<\infty}{\text { u.b. }}\left\{(1+|x|)^{m}\left|f(x)-g_{\alpha}(x)\right|\right\} \rightarrow 0 \quad \text { as } \alpha \rightarrow \infty .
$$

COROLlaRY (ii) $\left({ }^{7}\right)$. There are functions $g_{\alpha}(x) \in G_{\alpha}(0<\alpha<\infty)$ such that, for any integer $m$,

$$
\underset{-\infty<x<\infty}{\text { u.b. }}\left\{(1+|x|)^{m}\left|x^{r}-g_{\alpha}(x)\right|\right\} \rightarrow 0 \quad \text { as } \alpha \rightarrow \infty,
$$

where $r>0$, arg $x=0(x>0)$ or arg $x=\pi(x<0)$.

Corollary (iii). If $f(x)$ is defined in $(0, \infty)$ and if $(x+i)^{m} f\left(x^{2}\right)$ is uniformly continuous of some order, then there are functions $\gamma_{\alpha}(z) \in G_{\alpha}^{(1 / 2)}(0<\alpha<\infty)$ such that

$$
\underset{0<x<\infty}{\text { u.b. }}\left\{(1+x)^{m / 2}\left|f(x)-\gamma_{\alpha}(x)\right|\right\} \rightarrow 0 \quad \text { as } \alpha \rightarrow \infty \text {. }
$$

Corollary (iv)( ${ }^{8}$ ). The result stated in Corollary (ii) holds when $G_{\alpha}$ is replaced by $G_{\alpha}^{(1 / 2)}$ and the interval $(-\infty, \infty)$ by $(0, \infty)$.

Corollary (v). Let $f(x)=\phi(x) \psi(x)$ where $\psi(z) \in G_{c}$ for some $c>0$ and $|\psi(x)|<A\left(1+|x|^{m}\right)$ for some $m \geqq 0$, while $(x+i)^{m} \phi(x)$ is uniformly continuous of some order. Then, uniformly in $(-\infty, \infty), f(x)$ can be approximated by elements $g_{\alpha}(x)$ of $G_{\alpha}(c<\alpha<\infty)$.

Example. $f(x)=x^{r} \sin x(0<r<\infty)$; here $\psi(z)=\sin z \in G_{1}, m=0$.

Remark to Theorem 3. If $f(x)$ is merely supposed to be $O\left(|x|^{n}\right)$ for $x \rightarrow \pm \infty$ the condition is not necessary; take, for instance, $f(x)=x \sin x$. Certainly it is necessary that $(x+i)^{-n} f(x)$ should be uniformly continuous of order one. This condition, however, is not sufficient; take, for instance, $n=2$, $f(x)=x \sin x^{2}, m=0$.

Corollary (ii) is evident for the special case when $m$ is fixed, the $g_{\alpha}(z)$ depending on $m$. The general case is then deduced by the diagonal method, if we take $m \rightarrow \infty$.

Under the hypothesis of Corollary (iii), we have by Corollary (i)

$$
\underset{-\infty<y<\infty}{\text { u.b. }}\left\{(1+|y|)^{m}\left|f\left(y^{2}\right)-g_{\alpha}(y)\right|\right\} \rightarrow 0 \quad \text { as } \alpha \rightarrow \infty .
$$

This holds when $y$ is replaced by $-y$. Taking $y^{2}=x, 2^{-1}\left\{g_{\alpha}(y)+g_{\alpha}(-y)\right\}$

(7) Compare Theorem $11 \mathrm{G}$.

(8) Compare Theorem 11' G. 
$=\gamma_{\alpha}(x)$, we arrive at the result required.

3.2. The necessity of the condition in Theorem 3, and its sufficiency in the case $m=0$. By (3.11), any of the functions $(x+i)^{m} g_{\alpha}(x)$ is uniformly bounded of order $n$. By Theorem 2, it is uniformly continuous of order $n$, so is, therefore, $f(x)$.

Throughout this section and the following ones, we take now

$$
\kappa(t)=c_{M}\left(\frac{\sin t /(2 M)}{t}\right)^{2 M},
$$

fixing the positive integer $M$ large enough for the requirements of the proof concerned, and fixing $c_{M}$ so that $\int_{-\infty}^{\infty} k(t) d t=1$. The function $\kappa(z)$ belongs to $G_{1}$. As $f(x)$ is supposed to be uniformly continuous of order $n$, the function

$$
\gamma_{\alpha}(z)=\alpha \int_{-\infty}^{\infty} \kappa\{\alpha(t-z)\} f(t) d t
$$

belongs to $G_{\alpha}$ by the Lemmas 5 and $5 \mathrm{~F}$. We have

$$
\gamma_{\alpha}(x)=\int_{-\infty}^{\infty} k(t) f(x+t / \alpha) d t .
$$

Hence

$$
\begin{aligned}
\int_{-\infty}^{\infty} \kappa(t) \Delta_{n, t / \alpha} f(x) d t & =\int_{-\infty}^{\infty} \kappa(t) \sum_{j=0}^{n}(-1)^{i} C_{n, j} f\left(x+\frac{n-j}{\alpha} t\right) d t \\
& =\sum_{j=0}^{n-1}(-1)^{i} C_{n, j} \gamma_{\alpha /(n-i)}(x)+(-1)^{n} f(x) .
\end{aligned}
$$

Denoting the sum on the right by $(-1)^{n-1} g_{\alpha}(x)$, we have $g_{\alpha}(z) \in G_{\alpha}$ : In consequence of Lemma 4, we have

$$
\left|\Delta_{n, t / \alpha} f(x)\right| \leqq A\left(1+|t|^{n}\right),
$$

uniformly with respect to $x$ and $t \quad(-\infty<x<\infty,-\infty<t<\infty)$ and to $\alpha .(1 \leqq \alpha<\infty)$. Hence, given $\epsilon>0$, we have

$$
\left|\left(\int_{-\infty}^{-N}+\int_{N}^{\infty}\right) \kappa(t) \Delta_{n, t / \alpha} f(x) d x\right|<\epsilon / 2
$$

for a suitable $N>0$. By the uniform continuity (order $n$ ) of $f(x)$, we have $\left|\Delta_{n, t / \alpha} f(x)\right| \leqq \epsilon / 2$ for $|t / \alpha| \leqq \delta=\delta(\epsilon)$, that is, for $|t| \leqq N$ when $\alpha \geqq N / \delta$. Hence

$$
\left|\int_{-N}^{N} \kappa(t) \Delta_{n, t / \alpha} f(x) d t\right| \leqq \frac{\epsilon}{2} \int_{-\infty}^{\infty} \kappa(t) d t=\frac{\epsilon}{2} \text {. }
$$

Therefore the modulus of the integral on the left of (3.22) is smaller than $\epsilon$, 
which completes the proof for the case $m=0$.

\subsection{Two lemmas.}

LEMMA 8. If $f(x)$ is uniformly continuous of order $n$, then also $(x+i)-i f(x)$ $(j=1,2, \cdots)$ is uniformly continuous of order $n$.

In consequence of Theorem 3 , with $m=0$, we have

$$
\underset{-\infty<x<\infty}{\text { u.b. }}\left|(x+i)^{-i} f(x)-(x+i)^{-i} g_{\alpha}(x)\right| \rightarrow 0 \quad \text { as } \alpha \rightarrow \infty .
$$

Now $f(x)$, therefore also $g_{\alpha}(x)$, is uniformly bounded of order $n$; hence, by Theorem $2, g_{\alpha}^{(n)}(x)$ is bounded in $(-\infty, \infty)$. Consequently

$$
\frac{d^{n}}{d x^{n}}\left(\frac{g_{\alpha}(x)}{(x+i)^{j}}\right)=\sum_{l=0}^{n} c_{l} \frac{g_{\alpha}^{(n-l)}(x)}{(x+i)^{j+l}}
$$

is bounded in $(-\infty, \infty)$, and so $(x+i)^{-i} g_{\alpha}(x)$ is uniformly continuous of order $n$ for any $\alpha$. So is, by (3.31), $(x+i)^{-i} f(x)$.

An elementary proof can be given for $j \geqq n / 2$. For $j \geqq n,(x+i)^{-i f} f(x)$ is uniformly continuous of order one, as we see by applying Theorem 1 to this function.

LEMMA 9. If (i) $\left|\Delta_{n, h} f(x)\right| \leqq K$ for $-H \leqq h \leqq H$ uniformly in $-\infty<x<\infty$ and (ii) the numbers $a_{0}, a_{1}, \cdots, a_{m}(m \geqq n)$ satisfy the $n$ conditions

$$
\sum_{j=0}^{m} j^{l} a_{j}=0 \quad\left(l=0,1, \cdots, n-1 ; j^{0}=1 \text { for } j=0\right),
$$

then

$$
\left|\sum_{j=0}^{m} a_{j} f(x+j h)\right| \leqq A K \quad(-H \leqq h \leqq H,-\infty<x<\infty),
$$

where $A$ depends on the $a_{j}^{\prime}$ 's only.

Let

$$
b_{j}=\sum_{s=j}^{m} a_{s} C_{s-j+n-1, n-1} \quad(j=0,1, \cdots, m) .
$$

From (3.32) we can deduce that $b_{0}=b_{1}=\cdots=b_{n-1}=0$; for $j>m$ we take $b_{j}=0$. Computing the $a_{j}$ 's, we obtain

$$
\begin{aligned}
a_{j} & =\sum_{s=0}^{n}(-1)^{\circ} C_{n, s} b_{j+s} \quad(j=0,1, \cdots, m), \\
\sum_{j=0}^{m} a_{j} f(x+j h) & =\sum_{j=n}^{m} b_{j} \Delta_{n, h} f\{x+(j-n) h\} .
\end{aligned}
$$


Thus

$$
\left|\sum_{j=0}^{m} a_{j} f(x+j h)\right| \leqq K \sum_{j=n}^{m}\left|b_{j}\right|=A K \quad(-H \leqq h \leqq H,-\infty<x<\infty),
$$

which completes the proof. Using Lemma 4, we deduce from (3.35) that

$$
\left|\sum_{j=0}^{m} a_{i} f(x+j t)\right| \leqq A^{\prime}\left(1+|t|^{n}\right) \quad(-\infty<t<\infty,-\infty<x<\infty) .
$$

3.4. Proof of Theorem 3 for $m>0$. Without loss of generality we may take $m \geqq n$. We set $f_{j}(x)=(x+i)^{-i} f(x)$. The function

$$
\gamma_{\alpha}(x)=\int_{-\infty}^{\infty} \kappa(t) f(x+t / \alpha) d t=\int_{-\infty}^{\infty} \kappa(t)(x+i+t / \alpha)^{m} f_{m}(x+t / \alpha) d t
$$

belongs to $G_{\alpha}$ (see 3.21). Now we have

$$
(\xi+\tau)^{m}=\xi^{m}+\sum_{j=1}^{m}(-1)^{j+1} C_{m} \tau^{i}(\xi+\tau)^{m-i} .
$$

Hence

$$
\gamma_{\alpha}(x)=(x+i)^{m} h_{\alpha}(x)+\sum_{j=1}^{n-1} \frac{c_{j}}{\alpha^{j}} h_{\alpha, j}(x)^{\cdot}+\sum_{j=n}^{m} \frac{c_{j}}{\alpha^{j}} h_{\alpha, j}(x),
$$

where

$$
\begin{array}{r}
h_{\alpha, j}(x)=\int_{-\infty}^{\infty} t^{i} k(t) f_{j}(x+t / \alpha) d t ; \quad h_{\alpha}(x)=\int_{-\infty}^{\infty} \kappa(t) f_{m}(x+t / \alpha) d t ; \\
c_{j}=(-1)^{i+1} C_{m, j} ; j=1,2 ; \cdots, m .
\end{array}
$$

For $j \geqq n$ the functions $h_{\alpha, j}(x)$ are bounded uniformly with respect to $x$ and $\alpha$, as $f_{j}(x)$ is bounded for $j \geqq n$. Hence the last term in (3.42) tends to zero as $\alpha \rightarrow \infty$, uniformly in $-\infty<x<\infty$; we denote it by $R_{\alpha}(x)$. When $n=1$ there is no second term on the right in (3.42). Let now $n \geqq 2$. We form

$$
\begin{aligned}
\sum_{s=0}^{2 n-2}(-1)^{s} C_{2 n-1,8} \gamma_{\alpha /(2 n-8-1)}(x) & =(x+i)^{m} \sum_{s=0}^{2 n-2}(-1)^{s} C_{2 n-1, s} h_{\alpha /(2 n-s-1)}(x) \\
+ & \sum_{j=1}^{n-1} \frac{c_{j}}{\alpha^{j}} \sum_{i=0}^{2 n-2}(-1)^{\circ} C_{2 n-1,8}(2 n-1-s)^{j} \\
& \cdot \int_{-\infty}^{\infty} t^{i} k(t) f_{i}\left(x+\frac{2 n-s-1}{\alpha} t\right) d t+\Im_{\alpha}(x),
\end{aligned}
$$

denoting by $\Im_{\alpha}(x)$ some finite linear form of the functions $R_{\alpha /(2 n-1-s)}$; evidently $\Im_{a}(x) \rightarrow 0$ as $\alpha \rightarrow \infty$, uniformly in $(-\infty<x<\infty)$. We set 


$$
\begin{aligned}
N & =2 n-1 ; \quad a_{s, j}=(-1)^{s} C_{N, s}(N-s)^{j} ; \\
\Phi_{j}(x, t) & =\sum_{s=0}^{N-1} a_{s, j} f_{j}\left(x+\frac{N-s}{\alpha} t\right)(j=1,2, \cdots, n-1) ; \\
H_{j}(x) & =\int_{-\infty}^{\infty} t^{j} \kappa(t) \Phi_{j} d t .
\end{aligned}
$$

We have

$$
\begin{aligned}
N>n>1, \quad a_{N, j}=0, \quad \Phi_{j}= & \sum_{\substack{c=0 \\
N}}^{N} a_{s, j} f_{j}\left(x^{\prime}+s t^{\prime}\right) \\
& \left(x^{\prime}=x+t N / \alpha, t^{\prime}=-t / \alpha\right),
\end{aligned}
$$

and

$$
\sum_{s=0}^{N} a_{s, j} s^{i}=0, \quad \text { for } l=0,1, \cdots, n-1
$$

and for any $j(j=1,2, \cdots, n-1)$; for the latter sum consists of the terms

$$
(-1)^{k} C_{j, k} N^{j-k} \sum_{s=0}^{N}(-1)^{*} C_{N, s} s^{k+l} \quad(k=0,1,2, \cdots, j)
$$

each of which can be shown to vanish, since $0 \leqq k+l \leqq 2 n-2=N-1$. For any of the $j$ 's concerned, therefore, the $a_{s, j}(s=0,1, \cdots, N)$ satisfy the conditions of Lemma 9 , and $f_{j}(x)$ is uniformly continuous of order $n$ by Lemma 8 . Hence, by (3.36), we have

$$
\left|\Phi_{i}\right| \leqq A_{j}\left(1+|t|^{n}\right) \quad(-\infty<t<\infty,-\infty<x<\infty)
$$

uniformly with respect to $x$ and $t$, and with respect to $\alpha$ for $1 \leqq \alpha<\infty$. Hence $H_{j}(x)$ is uniformly bounded with respect to $x$ and $\alpha$. Consequently the second term on the right in (3.44) tends to zero as $\alpha \rightarrow \infty$, uniformly with respect to $x$. Hence, taking

$$
g_{\alpha}(x)=\sum_{s=0}^{N-1}(-1)^{s} C_{N, s} h_{\alpha /(N-s)}(x)
$$

we have

$$
\underset{-\infty<x<\infty}{\text { u.b. }}\left|\sum_{\varepsilon=0}^{N-1}(-1)^{8} C_{N, 8} \gamma_{\alpha /(N-8)}(x)-(x+i)^{m} g_{\alpha}(x)\right| \rightarrow \quad \text { as } \alpha \rightarrow \infty .
$$

Now $f(x)$ is uniformly continuous of order $n$ and, therefore, of order $N$ as well. Thus the sum in (3.48) tends to $f(x)$ as $\alpha \rightarrow \infty$, uniformly with respect to $x$ (see 3.22, and so on). This completes the proof.

4. The half-plane. The results of $\$ 3$ will now be used to deal with func- 
tions which are analytic in a half-plane.

4.1. Results and lemmas.

THEOREM 4. If, for $0<y<\infty$, (i) $F(z)(z=x+i y)$ is analytic and (ii) $|F(z)|<A_{\delta} e^{\delta|z|}$ whenever $\delta>0$, and if (iii) $F(x)$, the limit-function of $F(z)$ $(y \rightarrow 0)$, is uniformly continuous of some order, then, given $m(m=0$, or $1,2, \cdots)$, there are functions $g_{\alpha}(z) \in G_{\alpha}(0<\alpha<\infty)$ such that

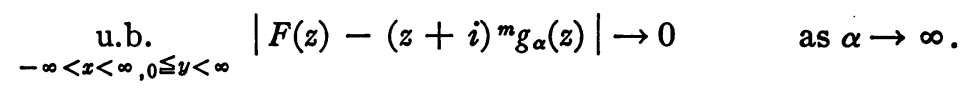

Corollary. If $F(z)$ satisfies (i) and (ii), and if (iii') for some integer $m>0,(x+i)^{m} F(x)$ is uniformly continuous of some order, then there are functions $g_{\alpha}(z) \in G_{\alpha}(0<\alpha<\infty)$ such that

$$
\underset{\alpha x<\infty, 0 \leq y<\infty}{\text { u.b. }}\left\{(1+|z|)^{m}\left|F(z)-g_{\alpha}(z)\right|\right\} \rightarrow 0 \quad \text { as } \alpha \rightarrow \infty .
$$

Evidently each of the functions $g_{\alpha}(z)$ nearly coincides with $F(z)$ for sufficiently large values of $z$, uniformly in the half-plane $y \geqq 0$. The application of the corollary to the function $z^{r}(r>0 ; 0 \leqq \arg z \leqq \pi)$ is obvious $\left({ }^{7}\right)$.

LEMma 10. If, for $0<\arg z<\pi / \alpha$, (i) $f(z)$ is analytic and (ii) $|f(z)|$ $<\exp \left(|z|^{\beta}\right)$ where $0<\beta<\alpha$, then $f(\xi)$, the limit-function of $f(z)(z \rightarrow \xi)$, exists for almost all $\xi$ when arg $\xi=0$ or arg $\xi=\pi / \alpha$. If, furthermore, (iii) $|f(\xi)| \leqq A$ uniformly on the two lines $\arg \xi=0$ and $\arg \xi=\pi / \alpha$, then $|f(z)| \leqq A$ uniformly for $0 \leqq \arg z \leqq \pi / \alpha$. The result holds if, instead of (ii), it is merely required that $|f(z)|<A_{\delta} \exp \left(\delta|z|^{\alpha}\right)$ for any $\delta>0$.

Lemma 11. If $F(z)$ satisfies the conditions (i) and (ii) of Theorem 4, and if $\left|\Delta_{n, h} F(x)\right| \leqq K$ for $-H \leqq h \leqq H,-\infty<x<\infty$, then

$$
\left|\Delta_{n, h} F(z)\right|=\left|\sum_{j=0}^{n}(-1)^{i} C_{n, j} F(z+j h)\right| \leqq K \quad(-H \leqq h \leqq H)
$$

and

$$
\left|\Delta_{n, t} F(z)\right| \leqq A\left(1+|t|^{n}\right) \quad(-\infty<t<\infty)
$$

uniformly in the half-plane $y \geqq 0$.

4.2. On the lemmas, and proof of the theorem. Lemma 10 is a slight generalization of well known results $\left({ }^{\circ}\right)$. Applying this result to the function $\Delta_{n, h} F(z)$, and using Lemma 4, we deduce Lemma 11.

To prove the theorem we show first that the functions $g_{\alpha}(z)$ are uniformly

(9) For example, E. C. Titchmarsh, The theory of functions, Oxford, 1932, 5.61 and 5.62. By the transformation $z=\bar{z}^{1 / \alpha}$ the lemma is reduced to its special case $0<\arg z<\pi, 0<\beta<1$. The function $\phi(z)=\exp \left(-\epsilon^{-i \pi \gamma / 2} z^{\gamma}\right) f(z)(\epsilon>0 ; \beta<\gamma<1)$, analytic and bounded for $y>0$, is now discussed using the Fatou theorem and the Poisson integral. 
bounded in the half-plane $y \geqq 0$, assuming that $m$ is not smaller than $n$, the order of uniform continuity of $F(x)$. We use the notations of $\$ 3.4$. The function $f_{m}(z)=(z+i)^{-m} F(z)$ is bounded for real values of $z$ and, by Lemma 10, therefore, uniformly bounded for $y \geqq 0$. As $\tau^{2} \kappa(\tau)$ is uniformly bounded for $-\alpha y \leqq \Im(\tau)<0\left({ }^{10}\right)$, we may apply the Cauchy theorem to the integral

$$
h_{\alpha}(z)=\alpha \int_{-\infty}^{\infty} \kappa(\alpha t-\alpha z) f_{m}(t) d t=\int_{-\alpha i y-\infty}^{-\alpha i \nu+\infty} \kappa(\tau) f_{m}\left(\frac{\tau}{\alpha}+z\right) d \tau,
$$

fixing $z(y>0)$. Hence

$$
\left|h_{\alpha}(z)\right|=\left|\int_{-\infty}^{\infty} \kappa(t) f_{m}\left(\frac{t}{\alpha}+z\right) d t\right| \leqq B \int_{-\infty}^{\infty} \kappa(t) d t=B,
$$

uniformly with respect to $z(y \geqq 0)$. Therefore the function $g_{\alpha}(z)$, defined by (3.47), is uniformly bounded for $y \geqq 0$. Thus we can apply Lemma 10 to the function $F(z)-(z+i)^{m} g_{\alpha}(z)$; and using (3.11), we have

$$
\underset{y \geq 0,-\infty<x<\infty}{\text { u.b. }}\left|F(z)-(z+i)^{m} g_{\alpha}(z)\right| \leqq \underset{-\infty<x<\infty}{\text { u.b. }}\left|F(x)-(x+i)^{m} g_{\alpha}(x)\right| \rightarrow 0
$$

as $\alpha \rightarrow \infty$. This completes the proof.

Added in proof, January 20,1947. If in the theorem or in Lemma 10, respectively, (ii) is replaced by (ii)': $|F(z)|$, or $|f(z)|$ (with $\alpha=1$ ) is majorised by $A_{\delta} \exp \{(c+\delta)|z|\} \quad(c>0)$, then (4.11) holds uniformly in any strip $0 \leqq y \leqq B$, or $|f(z)| \leqq A e^{c y}$ for $y>0$.

THE UNIVERSITY

Edgbastan, Birmingham, England

(10) G. P6lya and G. Szegö, Aufgaben und Lehrsätze aus der Analysis, vol. 2, Berlin, 1925,

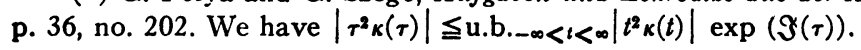

